\title{
Early Gastric Cancer with Juxta Regional Lymph Node Matastases
}

\author{
TAKAHO TANAKA, JINRYO TAKEDA, KIKUO KOUFUJI, \\ KEN HASHIMOTO AND TERUO KAKEGAWA \\ Department of Surgery, Knrume University School of Medicine, \\ Kurume, 830 Japan
}

\author{
Received for publication September 4, 1991
}

\begin{abstract}
Summary: During the 12 years from 1977 to 1988, a total of 425 cases of early gastric cancer (EGC) were treated by resection. Of these, $53(13 \%)$ had lymph node metastases, occurring in 6 cases of mucosal cancer (m-cancer) and 47 cases of submucosal cancer (sm-cancer). Among the patients with positive lymph node metastases, $46(87 \%)$ had metastases in the Group 1 perigastric regional nodes, and $8(2 \%)$ had metastases in Group 2 lymph node. These date support the general view that lymph node metastasis occurs in only $2-5 \%$ of $\mathrm{m}$-cancers and $15-20 \%$ of sm-cancers, and then mainly in Group 1 lymph nodes. A rare case of early cancer with Group 3 juxta regional lymph node metastasis $\left(n_{3}+\right)$ is described in this report with special attention to the surgical management and postoperative chemotherapy.
\end{abstract}

Key words: early gastric cancer - juxta lymph node - matastasis - hepatoduodenal ligament - chemotherapy

\section{Introduction}

The prognosis for gastric cancer has improved year by year because of the recent increased detection rate of early gastric cancer due to mass screening examinations, together with advances in radiographic, endoscopic, biopsy, operative techniques and postoperative chemotherapy.

Early gastric cancer (EGC) is defined as a carcinoma confined to the mucosa or submucosa of the stomach, with or without lymph node metastasis. However, the lymph node metastasis rate is generally $2-5 \%$ in $\mathrm{m}$-cancer and $15-20 \%$ in smcancer, with the lymph node metastases observed mainly in the Group 1 lymph nodes in both types of cancer. A rare case of early gastric cancer with positive lymph node metastasis in the hepatoduodenal ligament of the Group 3 lymph nodes is described.

\section{Patients and Methods}

During the 12 years from 1977 to 1988 , a total of 425 cases of EGC were resected in the First Department of Surgery, Kurume University Hospital. Of these, 53 (12.5\%) had lymph node metastases. Lymph node metastases were found in 6 cases of $\mathrm{m}$-cancer and in 47 cases of smcancer. Among the EGC with positive lymph node metastases, 46(87\%) were found in the Group 1 lymph nodes. Lymph node metastases in Group 2 nodes occurred in $8(2 \%)$ of 425 cases. EGC with lymph node metastases in the Group 3 nodes occurred in only one $(0.2 \%)$ of 425 cases and that metastasis was found in the hepatoduodenal ligament.

A fifty-eight year old female patient underwent distal gastrectomy with groups 1 and 2 lymph node dissection for a superficial depressed type of IIc in the antrum. During the operation, an enlarged 
lymph node on the left side of the hepatoduodenal ligament was found, and a lymph node dissection along the hepatoduodenal ligament was performed. Figure 1 shows the resected specimen with a tumor, $80 \times 65 \mathrm{~mm}$ in size. Histologically, the cancer was a signet ring cell carcinoma with a cancer depth within the submucosa (sm-cancer) of the stomach and superficially invading the duodenum (Figs. 2 and 3 ).

Figure 4 is a hematoxin-eosin stained section of the metastasized lymph node

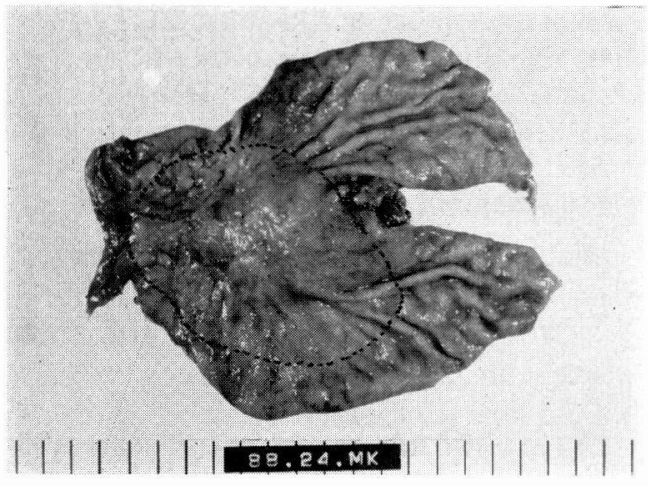

Fig. 1. The resected specimen of the early gastric cancer type IIc in the antrum with invasion of the duodenum. The tumor size was $80 \times 65 \mathrm{~mm}$.

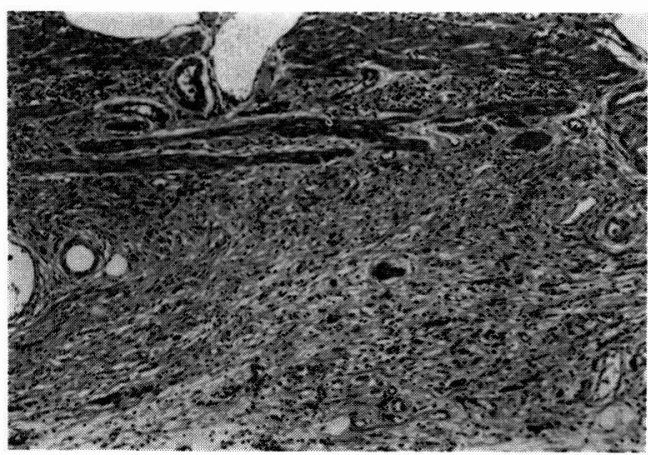

Fig. 3. Invasion of the signet ring cell carcinoma. Proliferation of the connective tissue can be seen in the submucosa of the stomach $(\mathrm{HE} \times 100)$. in the hepatoduodenal ligament (Group 3) that confirmed the histological type to be the same as that of the primary gastric cancer. Lymph node metastases were also confirmed along the lesser curvature (Group 1) and infrapyloric node (Group 1).

No postoperative complications occurred. Postoperative chemotherapy was administered for 2 years using MMC, intravenously, and $5-\mathrm{Fu}$, orally. The patient is now disease-free after a follow-up of 3 years and 6 months.

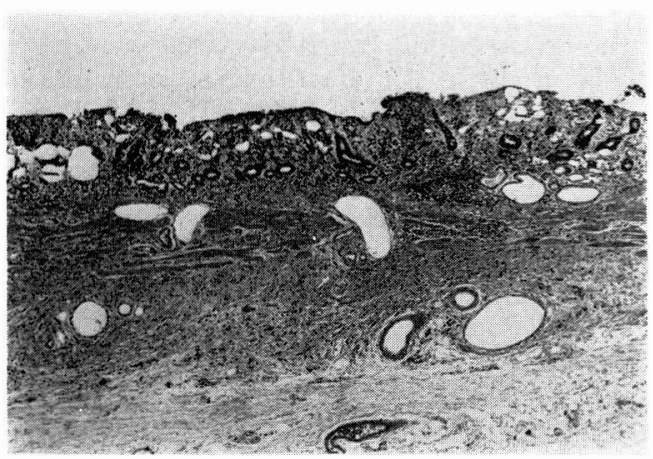

Fig. 2. Signet ring cell carcinoma in the mucosa and submucosa of the stomach. $(\mathrm{HE} \times 20)$.

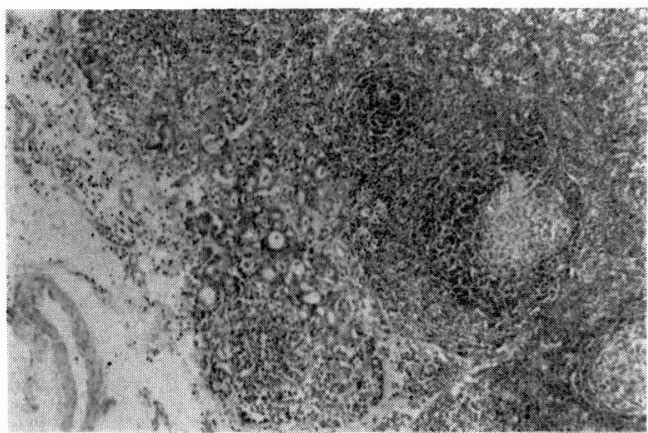

Fig. 4. Metastatic lymph onde in the hepatoduodenal ligament. The signet ring cell carcinoma can be seen in the marginal sinus of the lymph node $(\mathrm{HE} \times 100)$. 


\section{Discussion}

Early gastric cancer is defined as a cancer confined to the mucosa and submucosa of the stomach with or without lymph node metastasis. Lymph node metastasis in EGC can be correlated with the depth of cancer invasion into the submucosa and to the tumor size. While the incidences of $\mathrm{m}$-cancer and sm-cancer are almost equal in recent Japanese studies (Endo and Habu, 1990); the lymph node metastasis rate in $\mathrm{m}$-cancer is low, 2-5\% (Fujita et al. 1983; Abe et al. 1984), compared with a rate of $20 \%$ for smcancer. However, preoperative diagnosis of lymph node metastasis is difficult. A rare case of EGC with positive lymph node metastasis in the Group 3 lymph nodes is reported.

$\mathrm{R}_{2}$-gastric resection which involves gastrectomy, omentectomy and the complete removal of Groups 1 and 2 regional lymph nodes has been gererally accepted as the procedure of choice for the treatment of EGC. As a result, surgical treatment for EGC patients has achieved an excellent 5 year survival rate of about $97 \%$ (Kitaoka et al. 1984; Takeda et al. 1987). Habu et al. (1986) reported that cancer recurrences occurred more of ten in patients who had undergone no lymph node dissection $(9.4 \%)$ than in those who had undergone lymph node dissection (1.5 \%). The cancer recurrence pattern in EGC is mainly hematogenous metastasis to the liver (Inokuchi, 1984). Kaibara et al. (1984) reported that both cancer recurrences and cancer deaths were highest for the mixed-macroscopic type, such as $\Pi \mathrm{a}+\Pi \mathrm{c}$, of sm-cancer with positive lymph node metastasis. In the present study, almost all the lymph node metastases were found in the Group 1 lymph nodes (87\%). Lymph node metastases in the Group 2 nodes occurred in only 8 (2\%) of the 425 cases. EGC with a positive lymph node metastasis in the Group 3 nodes $\left(\mathrm{n}_{3}+\right)$ occurred in only one $(0.2 \%)$ of the 425 cases. Ohta et al. (1987) analyzed 1003 cases of EGC and found four $\mathrm{n}_{3}(+)(0.4 \%)$. Ishii et al. (1981) has reported two $\mathrm{n}_{3}(+)$ cases out of 128 EGC (1.6\%), and Murakami (1979) reported one EGC with $\mathrm{n}_{3}(+)(2 \%)$ in $57 \mathrm{sm}$-cancers.

There has been no report of an $\mathrm{m}$ cancer with $\mathrm{n}_{3}(+)$ metastasis. Of the seven known cases of sm-EGC with $\mathrm{n}_{3}$ $(+)$, the cancer was most frequently observed in the antrum with the positive lymph nodes in the hepato-duodenal ligament. There have been two reports of positive para-aortic lymph node metastases $\left(\mathrm{n}_{4}+\right)$ in EGC by Hirono et al. (1984) and by Ohta et al. (1987).

Murakami (1979) reported that the 5 year survival rate for EGC with $\mathrm{n}_{3}(+)$ was $25 \%(1 / 4)$. In the present report on an sm-cancer with $\mathrm{n}_{1}(+)$ and $\mathrm{n}_{3}(+)$ metastases, the survival is 3 years and 6 months to date.

It can be concluded that intraoperative macroscopic observation is important to find positive lymph node metastases even in cases of EGC, especially for the mixed-macroscopic type, sm-cancer and for the wide-spreading type EGC. When any lymph node metastasis is histologically positive, postoperative adjuvant chemotherapy should be administered even for patients with EGC who had undergone a curative operation. Moreover, aggressive lymph node dissection and postoperative adjuvant chemotherapy are needed for $\mathrm{n}_{3}(t)$ and $\mathrm{n}_{4}(+)$ cases of EGC with a similar approach to that for much advanced gastric cancer.

\section{References}

Abe, S., Ogawa, U., Nagasue, N., Sasaki, Y., Akamizu, H., Hirose, S., Yukaya, H. and SueniRo, S. (1984). Early gastric cancer: Results in a general hospital in Japan. World 
J. Surg. 8, 308-314.

Endo, M. and Habu, H. (1990). Clinical studies of early gastric cancer. Hepato-gastroenterol. 37, 408-410.

Fujita, Y., Nishioka, B., Sakiya, M., Kojima, O., Nomiyama, S., Oughi, T., Yamane, T., Kasuga, M. and MajIMA,S. (1983). Conservative surgery for regional lymphadenectomy in the treatment of early gastric carcinoma. Jpn. J. Surg. 13, 184-190.

Habu, H., Takeshita, K., Sunagawa, M. and Endo, M. (1986). Lymph node metastasis in early gastric cancer. Int. Surg. 71, 244-247.

Hirono, M., Suehiro, S., Hirai, T., Niimoto, M. and HATTORI, T. (1984). Early gastric cancer with widespread lymph node metastasis; A case report. Jpn. J. Surg. 14, 143-145.

INокUCH, K. (1984). Early gastric cancer, viewed from its growth patterns. Presented at the 8th world congress of the CICD (1984). Amsterdam, September 12, 1-34.

Ishil, T., Miura, T., Harada, T., Nakayama, H., Hirano, T., Yoshida, C., Nogawa, T., Hashimoto, S., FuKi, R., TAKaki, T., IshikaWa, Y., Kotake, Y.,
Shimotama, Y., Uchida, Y. and Tsuji, Y. (1981). The clinical consideration on the surgical treatment for early gastric cancer. Jpn. J. Gastroenterol. Surg. 14, 39-44.

Kaibara, N., Tamura, H. and Koga, S. (1984). Causes of death after surgery for early gastric cancer. Stomach and Intestine 19, 739743.

Kitaoka, H., Yoshikawa, K., Hirota, T. and ItABASHI, M. (1984). Surgical treatment of early gastric cancer. Jpn. J. Clin. Oncol. 14, 283-293.

Murakami, T. (1979). Early gastric cancer. World J. Surg. 3, 685-692.

Ohta, H., $\quad$ Noguchi, Y., T Takagi, K., Nishi, M., Kajitani, T. and Kato, Y. (1987). Early gastric cancer with special reference to macroscopic classification. Cancer 60, 1099-1106.

Takeda, J., Hashimoto, K., Machi, J., Hirai, Y., Yoshida, T., Ohmori, Y. and Kakegawa, T. (1987). Clinical and pathological evaluation of early gastric cancer and lymph node metastasis. Kurume Med. J. 34, 183-191. 